\title{
CARACTERIZAÇÃO DE PLACAS DE CIRCUITO IMPRESSO POR MICROSCOPIA ELETRÔNICA DE VARREDURA VISANDO RECUPERAÇÃO DE METAIS*
}

Marcos Paulo Kohler Caldas ${ }^{1}$

Viviane Tavares de Moraes $^{2}$ Jorge Alberto Soares Tenório ${ }^{3}$ Denise Crocce Romano Espinosa ${ }^{4}$

\section{Resumo}

A recuperação de metais presentes em equipamentos eletroeletrônicos tem atraído interesse em virtude de novas e diferentes tecnologias que visam a reutilização desses elementos químicos em novos processos produtivos. A recuperação de metais das placas de circuito impresso tem atraído o interesse das empresas, pois, ao mesmo tempo que produz bons resultados do ponto de vista econômico, também se pratica o desenvolvimento sustentável, reduzindo a extração de metais da natureza. Este trabalho tem como objetivo caracterizar as placas de circuito impressos (PCl's) de memória de computadores, através de imagens de microscopia eletrônica de varredura com espectroscopia por dispersão de energia (MEV/EDS), a fim de verificar a presença de metais, visando a sua recuperação.

Palavras-chave: Reciclagem; Recuperação de metais; Caracterização.

\section{CIRCUIT BOARDS PRINTED CHARACTERIZATION BY SCANNING ELECTRON MICROSCOPY AIMING RECOVERY OF METALS}

\section{Abstract}

The recovery of metals from electronic equipment has attracted interest because of new and different technologies that aim to reuse these chemicals elements in production processes. The recovery of metals from printed circuit boards is interesting to the companies because at the same time produces good results from the economic point of view, and promotes a sustainable development through the reduction extraction of nature metal. This work aims to characterize the printed circuit boards (PCl's) from computer memory through scanning electron microscopy and spectroscopic energy dispersive images (SEM / EDS) in order to verify the presence of metals, aiming the recovery of metals.

Keywords: Recycling; Recovery of metals; Characterization.

Doutorando em Engenharia Química, Escola Politécnica da USP.

Pós Doutoranda em Engenharia Química, Escola Politécnica da USP, São Paulo, SP - Brasil.

Professor Titular do Dept. Eng. Química, Escola Politécnica da USP.

Professora Associada do Dept. Eng. Química, Escola Politécnica da USP. 


\section{INTRODUÇÃO}

O consumo desenfreado e o ritmo acelerado da tecnologia trazem como consequência a obsolescência programada, que, aliada com o fim da vida útil e ocorrências de defeitos, transformam os equipamentos eletroeletrônicos em sucatas eletrônicas [14]. A geração crescente deste resíduo tem evoluído para um problema ambiental, onde a preocupação está relacionada à lista de elementos químicos contidos nas sucatas eletroeletrônicas (vários tóxicos tais como: mercúrio, chumbo, arsênio), e a disposição incorreta desses resíduos[5,6].

Quando as sucatas são dispostas em aterros sanitários, por exemplo, agrava-se ainda mais a situação; pois, com a disposição de elementos químicos que sofrem processo de lixiviação provocado pelos ácidos graxos (provenientes da degradação da matéria-orgânica), pode ocorrer a contaminação do solo e água subterrânea [7].

A exposição do resíduo metálico no meio ambiente pode sofrer alterações tais como: densidade, toxidade, dentre outras propriedades físico-químicas, proporcionando o desequilíbrio ecológico [8]. Para evitar a contaminação de áreas e recursos naturais provocadas pela disposição desses resíduos, diversas alternativas vem sendo aplicadas, umas delas é a minimização dos impactos ambientais através da reciclagem e recuperação dos metais preciosos presentes nas sucatas tecnológicas $[9,10]$.

Recuperar metais preciosos a partir de resíduos de placas de circuito impresso ( $\mathrm{PCl}$ ) tornou-se algo de interesse das empresas, já que os metais preciosos e o cobre representam mais de $95 \%$ do valor total intrínseco da placa, além de sua concentração no resíduo ser de até 10 vezes maior que nos minérios [11-13]. Para a empresa, é a oportunidade de praticar o desenvolvimento sustentável adquirindo matéria-prima, que se encontra escassa na natureza, a partir de um resíduo [14,15]. O desenvolvimento sustentável é uma das motivações para a prática de reciclagem de equipamentos eletroeletrônicos obsoletos. Três dos desafios da gestão de resíduos de equipamentos eletroeletrônicos (REEE) são exatamente o tripé da sustentabilidade (preservação do meio ambiente, reuso e remanufatura de REEE e inclusão dos catadores gerando emprego e renda, e também uma forma da empresa fazer marketing ambiental)[16].

Os REEE, tem se tornado objeto de pesquisas [17-19] com intuito de minimizar os riscos à saúde e ao meio ambiente. A preocupação está relacionada aos elementos presentes nas placas de circuito impresso (PCls) dos equipamentos eletroeletrônicos (EEE), doze deles são altamente prejudiciais ao meio ambiente e, consequentemente, à saúde das pessoas, entre eles estão o chumbo, mercúrio e o cádmio. Além disso, os REEE possuem metais preciosos como ouro, prata, e a platina; metais que tem incentivado o mercado de reciclagem [20].

A recuperação dos metais preciosos presentes nas placas de circuito impresso retém boa parte dos estudos sobre sua reciclagem $[3,5,6]$. O interesse na obtenção destes metais é devido ao maior valor agregado em relação aos demais. Como mostra a Tabela 1, o valor referente aos metais preciosos pode chegar a mais de $70 \%$ do valor gasto com metais na produção da placa. 
Tabela 1 - Composição e o valor intrínseco dos metais presentes em placas de circuito impresso, adaptado de [21]

\begin{tabular}{llll}
\hline Metal & \%Massa & Valor(£/Kg) & Valor \% \\
\hline Ouro & 0.025 & 14200 & 65.4 \\
Paládio & 0.01 & 6200 & 11.4 \\
Cobre & 16 & 3.3 & 9.7 \\
Prata & 0.1 & 250 & 4.6 \\
Estanho & 3 & 8.1 & 4.5 \\
Níquel & 1 & 13.2 & 2.4 \\
Alumínio & 5 & 1.2 & 1.1 \\
Chumbo & 2 & 1.3 & 0.5 \\
Zinco & 1 & 1.2 & 0.2 \\
Ferro & 5 & 0.1 & 0.1
\end{tabular}

O valor agregado destes metais sugere a necessidade da reciclagem das placas de circuito impresso. A recuperação destes metais pode ser uma alternativa na obtenção de matéria-prima para a síntese de nanopartículas metálicas, que são utilizadas cada vez mais na indústria, acarretando em maior quantidade de estudos sobre nanotecnologia.

\section{MATERIAIS E MÉTODOS}

Foram avaliadas PCl's de memória de computadores obsoletos

\subsection{Processamento físico}

Foram utilizadas aproximadamente $5 \mathrm{~kg}$ de placas de memória para esta pesquisa.

\subsubsection{Cominuição}

No processamento físico, foi utilizado o moinho de facas (marca Rone, modelo FA2305) com grelha de $9 \mathrm{~mm}$, seguido de moagem em moinho de martelos (marca Astecma, modelo MDM 18/18) com grelha de $2 \mathrm{~mm}$, tendo como finalidade a redução do tamanho, aumento da área superficial e liberação dos materiais das placas de circuito impresso, facilitando a recuperação de metais [22]. Após a moagem foi feito o quarteamento, visando melhor homogeneização das amostras, até obtenção de frações de $20 \mathrm{~g}$.

\section{Caracterizacão}

\subsubsection{MEV/EDS}

Para os ensaios de MEV/EDS, a placa de memória foi cortada em locais distintos, com o objetivo de visualizar a distribuição dos elementos presentes, assim como o número de camadas de cobre. Os pontos foram definidos de forma a abranger a maior diversidade de componentes presentes na amostra. O MEV utilizado foi da marca Phenom modelo proX.

Todas as amostras cortadas foram embutidas em resina epóxi.

\subsubsection{Análise em lupa binocular}

Através desse ensaio pode-se verificar exposição da superfície metálica dos componentes das PCls. 
Após um corte na PCI para exposição de sua composição, realizou-se a observação da seção transversal da placa, identificando-se o número de camadas de cobre presente.

A lupa utilizada no ensaio foi da marca Zeiss modelo stemi 2000-C.

\section{RESULTADOS E DISCUSSÃO}

\subsection{Processamento físico}

As placas de memória desmanteladas manualmente tiveram alguns componentes segregados, como os dissipadores de alumínio, suportes dos processadores e parafusos, conforme Figura 1.
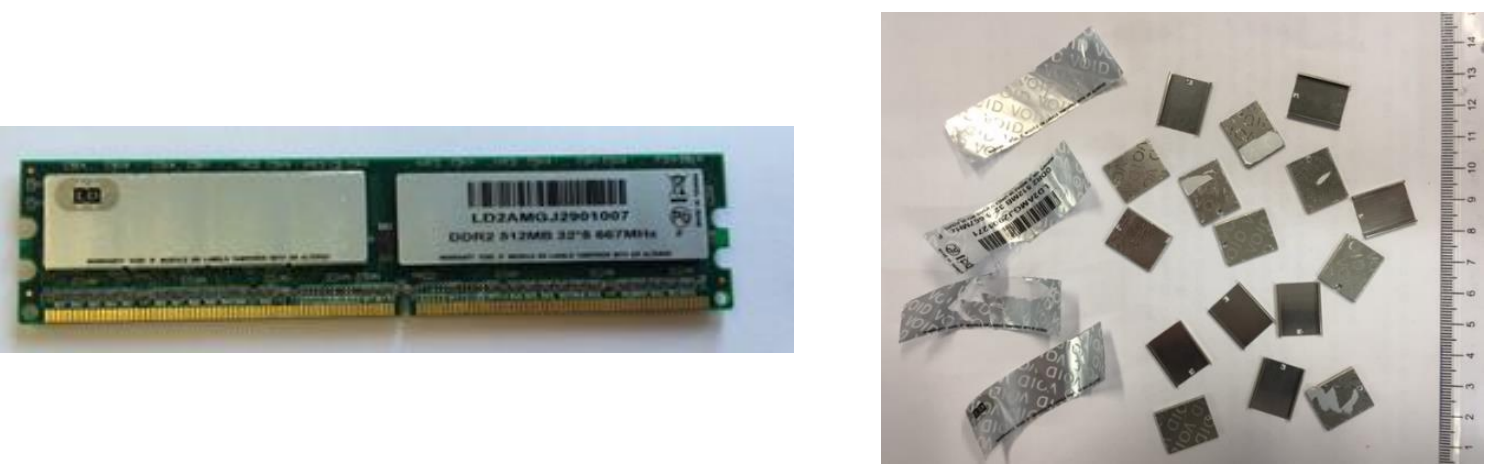

Figura 1-Placa de memória e componentes removidos dela por desmantelamento manual

A retirada desses componentes foi essencial para a obtenção de um substrato isento de peças soltas para obtenção de PCls mais similares possíveis.

As PCl seguiram para as etapas de cominuição (moagem em moinho de facas seguida de martelos), cujas perdas estão mostradas na Tabela 2.

As PCls de placas mãe cominuídas foram nomeadas de PCI-mem-M.

Tabela 2 - Balanço de massa das placas mãe e de memória após cominuição

\begin{tabular}{cll} 
Placa & Moagem & Perda $(\%)$ \\
\hline \multirow{2}{*}{ PCl-mem-M } & $9 \mathrm{~mm}$ & 0,17 \\
\cline { 2 - 3 } & $2 \mathrm{~mm}$ & 1,29 \\
\hline & Total & $\mathbf{1 , 4 6}$
\end{tabular}

A perda de massa total, para a amostra de placa $\mathrm{PCl}$-mem-M foi de 1,46\%. Valores da ordem de $25 \%$ e $30 \%$ de perdas foram obtidos por Moraes (2011) [5] e Yamane (2012) [24], respectivamente, utilizando equipamento similar. As perdas menores encontradas indicam uma melhor eficácia no processo de cominuição empregado no presente trabalho, evitando a perda de finos. Uma menor perda de massa no processo de cominuição garante a obtenção de uma amostra mais próxima da composição real da PCl. 


\subsection{Caracterização}

\subsubsection{Análise em lupa binocular}

Após a cominuição das amostras, realizou-se a observação em lupa binocular, para avaliação da liberação de materiais e da exposição da superfície metálica dos componentes das PCls.

A Figura 2 apresenta a vista transversal da placa de memória feita com lupa binocular. Pode ser visto que a placa de memória é do tipo multi-layer e possui 6 camadas de cobre intercaladas por fibra de vidro e resina, Figura 2.

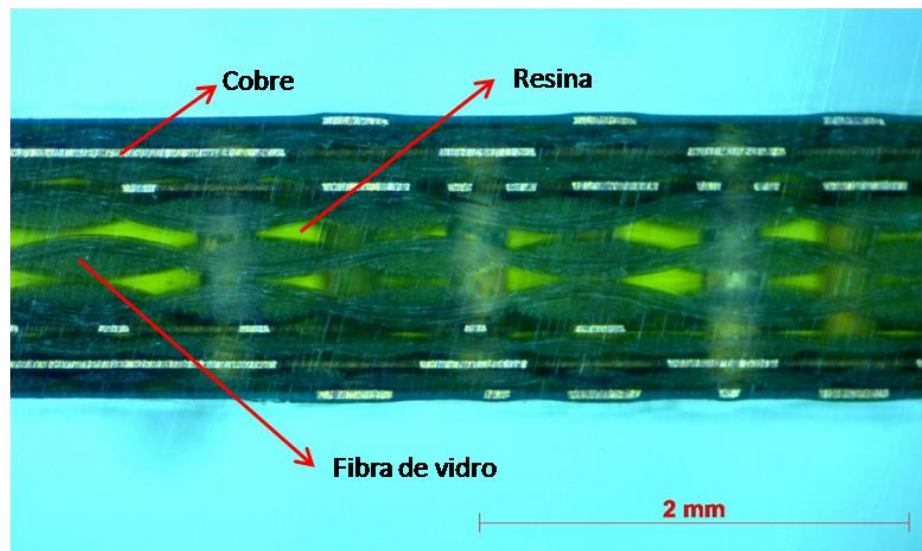

Figura 2 - Observação em lupa binocular da seção transversal da placa PCl-mem

As camadas que podem ser de cobre aparecem de forma pontilhada, não contínua, e representam as trilhas dos circuitos que não são paralelas a seção transversal da amostra analisada, muito comuns em PCls. As fibras de vidro podem ser identificadas como o material em formato ondular, caracterizado com camadas paralelas e perpendiculares a face da seção transversal, As paralelas podem ser vistas em formato senoidal e as perpendiculares se entrelaçam as paralelas formando ângulos de $90^{\circ}$.

A análise de lupa binocular não é conclusiva e, portanto, segue para análise de MEV/EDS a fim de confirmar os elementos visualizados na lupa.

\subsubsection{MEV/EDS}

A análise de MEV/EDS foi realizada nas amostras de PCI-mem, as amostras foram previamente embutidas para facilitar a visualização da seção transversal do substrato base das PCls, desta forma foi possível identificar as camadas de cobre, fibra de vidro e resina.

\subsubsection{MEV/EDS das PCls}

A Figura 3 apresenta a placa PCI-mem com os dois locais de amostragem para análise em MEV/EDS. Pode ser identificado na PCl-mem o local de retirada das amostras e sua respectiva posição após embutimento em resina. As amostras foram identificadas como amostragem A e amostragem B. 


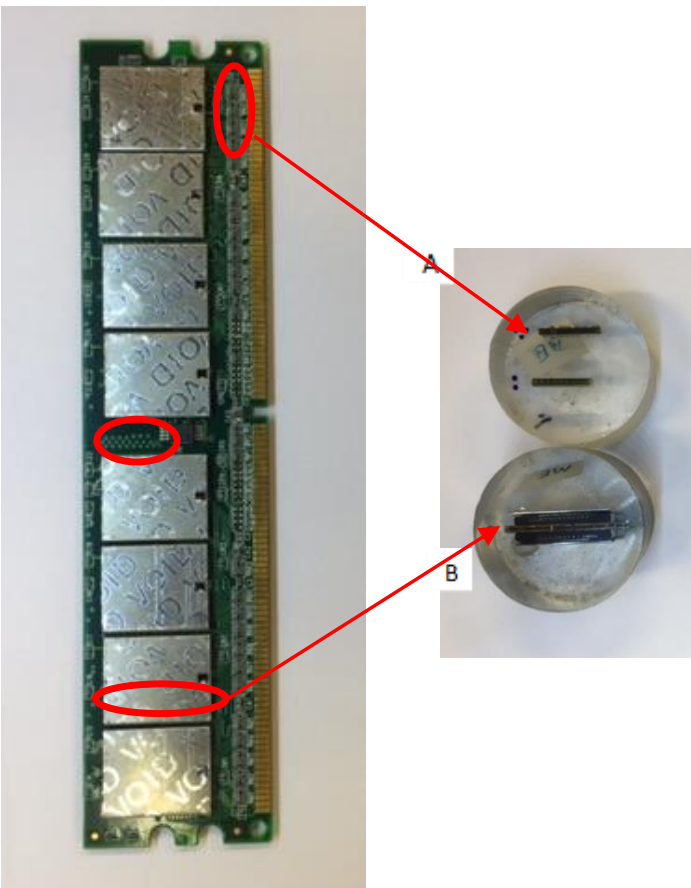

Figura 3 - Placa PCI-mem com localização dos pontos A, B e C de amostragem e identificação das amostras embutidas em resina.

\section{Amostragem A}

A posição desta amostragem foi definida em local contendo alguns componentes soldados à placa de memória. $\mathrm{O}$ objetivo da análise neste ponto é identificar a solda utilizada nos componentes SMD (Surface Mount Device), a disposição e quantidade de camadas de cobre e a composição do substrato da placa. A Figura 4 apresenta a imagem de lupa do ponto de amostragem A da placa PCI-mem e o detalhamento onde será feita a análise de MEV/EDS.
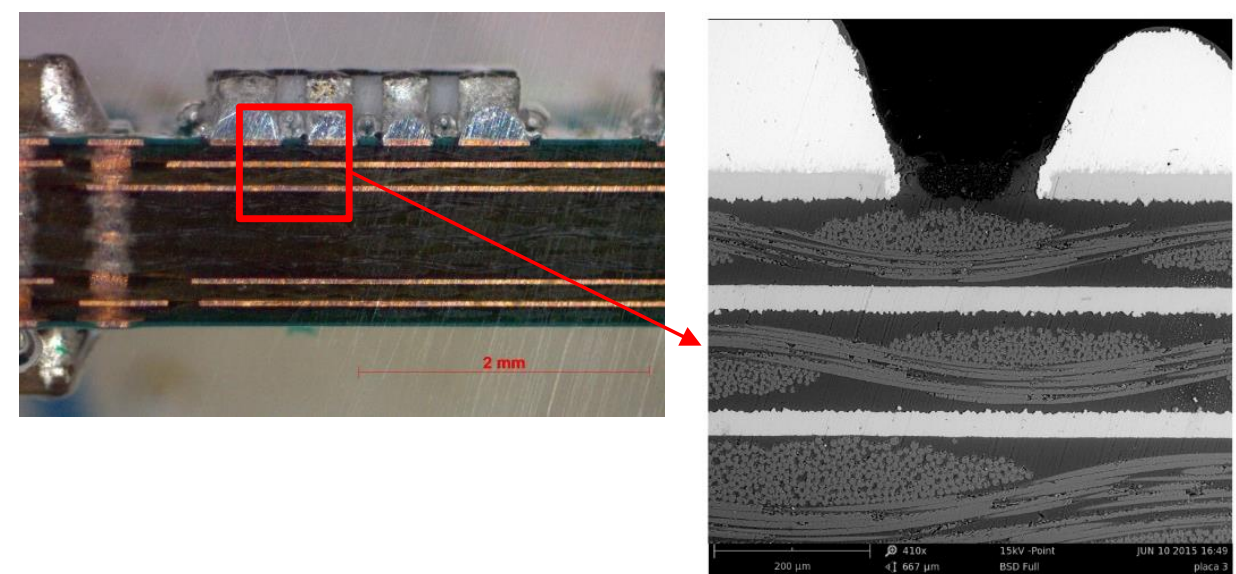

Figura 4-A direita, imagem de elétrons retro-espalhados da amostragem A da placa de memória. Á esquerda, detalhe mostrando a região em que a micrografia foi analisada.

A Figura 5 apresenta o mapeamento da área detalhada, a fim de se identificar os elementos presentes em sua composição. 


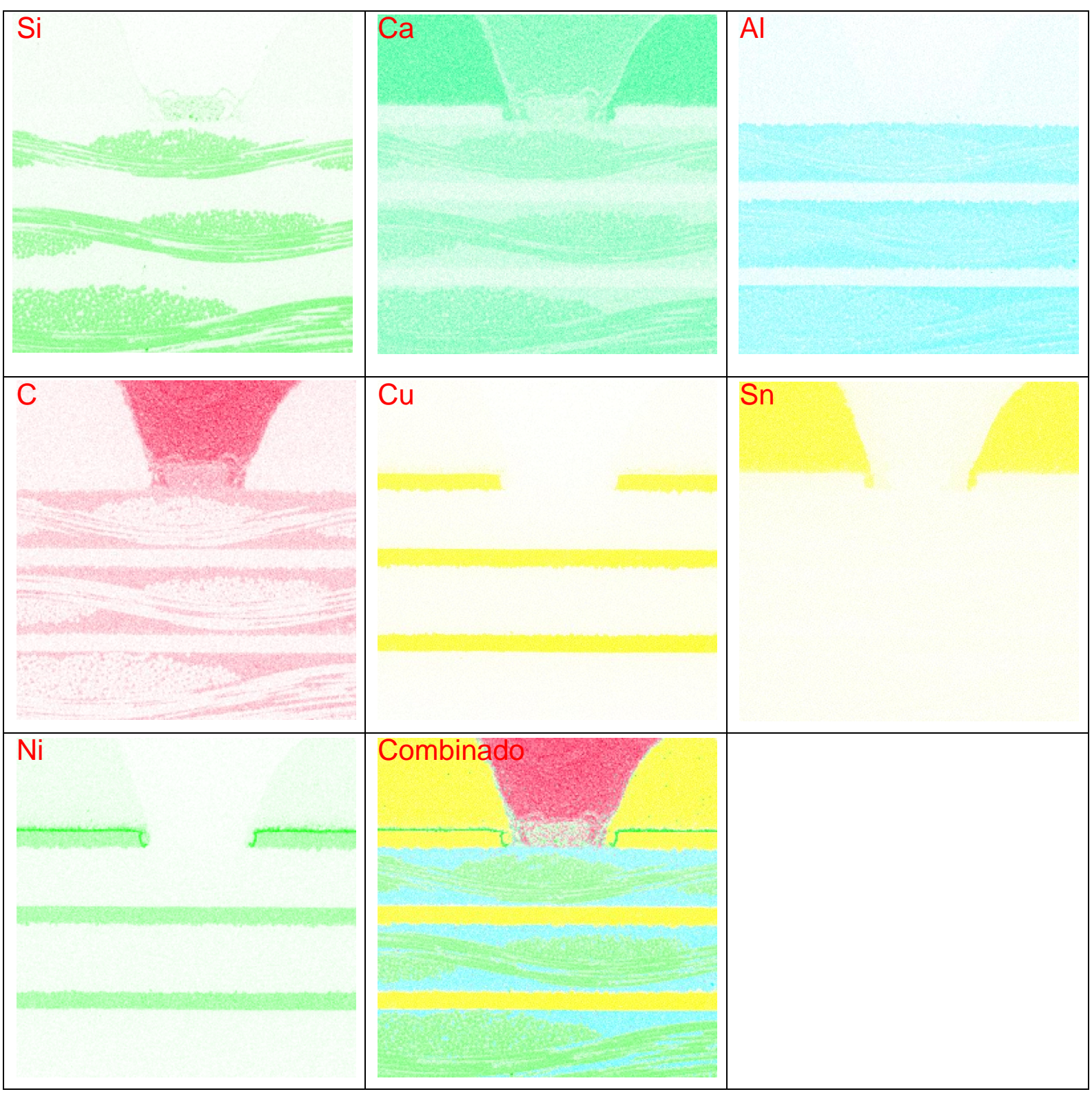

Figura 5 - Imagens de EDX da placa de memória, amostragem A

Pode-se verificar através da Figura 5 que a fibra de vidro da placa é composta de silício e cálcio, que a resina da placa é composta de alumínio e carbono. Verifica-se ainda que as trilhas são de cobre e que a solda não possui chumbo, já que é lead free, sendo composta basicamente de estanho. Nota-se também que entre a solda e as trilhas de cobre existe uma camada de níquel. O níquel é utilizado como uma barreira na metalização $\mathrm{Au} / \mathrm{Cu}$, devido compostos $\mathrm{Ni}-\mathrm{Sn}$ apresentarem baixas taxas de crescimento muito lenta e o Ni apresentar baixa taxa de difusão entre Au e Cu[25,26]. A Figura 6 apresenta o espectro de EDS em 4 pontos da amostragem a indicando a composição desses pontos. 


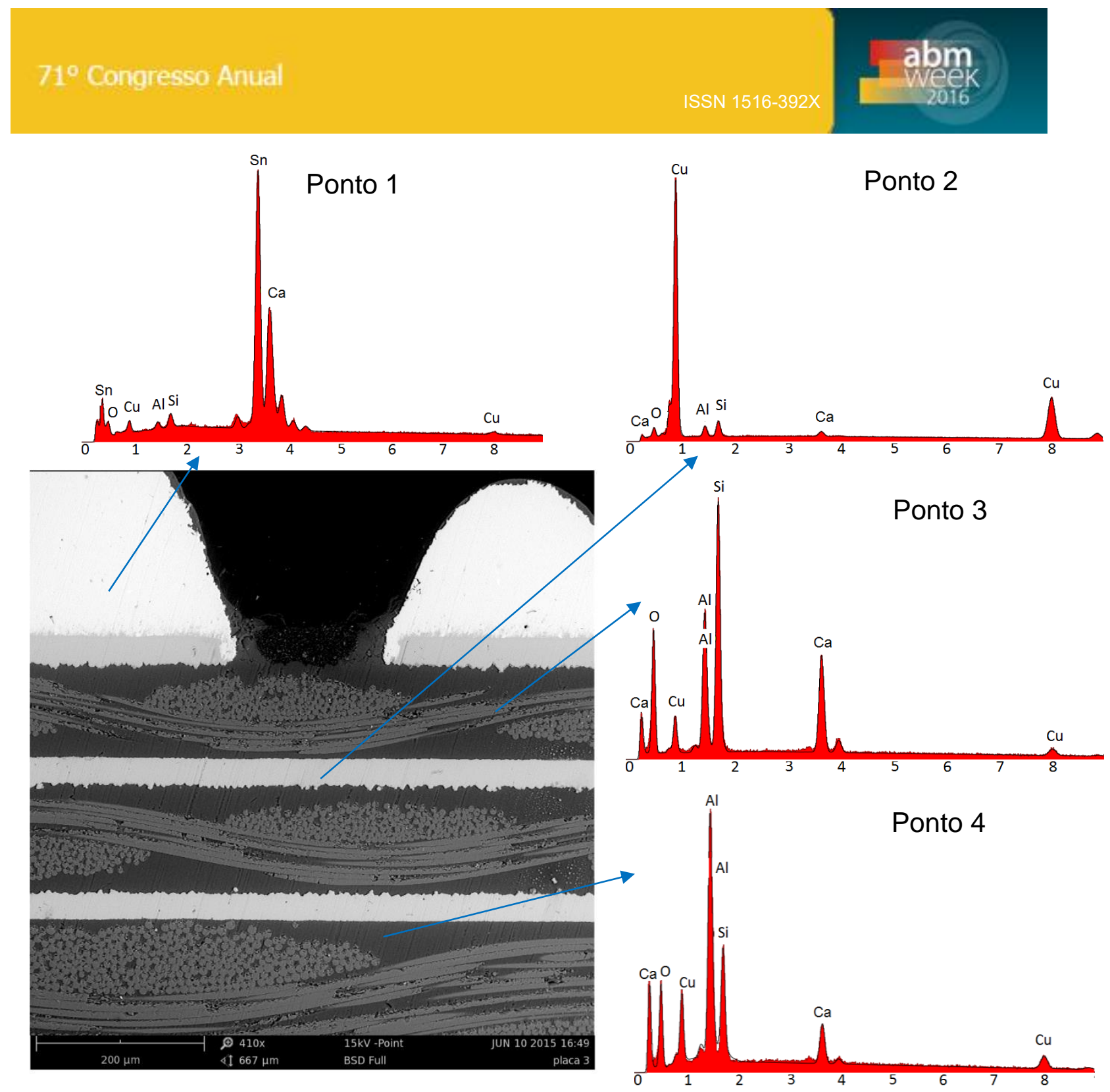

Figura 6 - Imagem de elétrons retro-espalhados da amostragem B da placa mãe e EDSs dos pontos; 1 (solda), 2 (trilha de cobre), 3 (fibra de vidro) e 4 (resina).

A Figura 6 mostra o espectro de EDS da amostragem A nos pontos 1, 2, 3 e 4. Pelo espectro de EDS do ponto 1 pode-se ver que a solda presente na placa de memória é composta de estanho e, possivelmente, lead free, pois não apresenta presença de chumbo. O espectro de EDS do ponto 2 mostra a camada de cobre da placa de memória. No espectro de EDS do ponto 3 e 4 pode-se verificar, novamente, que a fibra de vidro do substrato da placa de memória é composta de silício e cálcio e que entre as camadas de fibra de vidro existe uma resina composta de alumínio e carbono.

\section{Amostragem B}

A posição desta amostragem foi definida em local contendo circuitos integrados conectados a placa $\mathrm{PCl}-\mathrm{mem}$. $\mathrm{O}$ objetivo da análise neste ponto é verificar a composição dos circuitos integrados conectados a placa de memória e o substrato desses circuitos; e se as camadas de cobre e o substrato permanecem semelhantes as amostragens A e B. A Figura 7 apresenta a imagem de lupa do ponto de amostragem $\mathrm{C}$ da placa $\mathrm{PCl}$-mem e o detalhamento onde será feita a análise de MEV/EDS. 


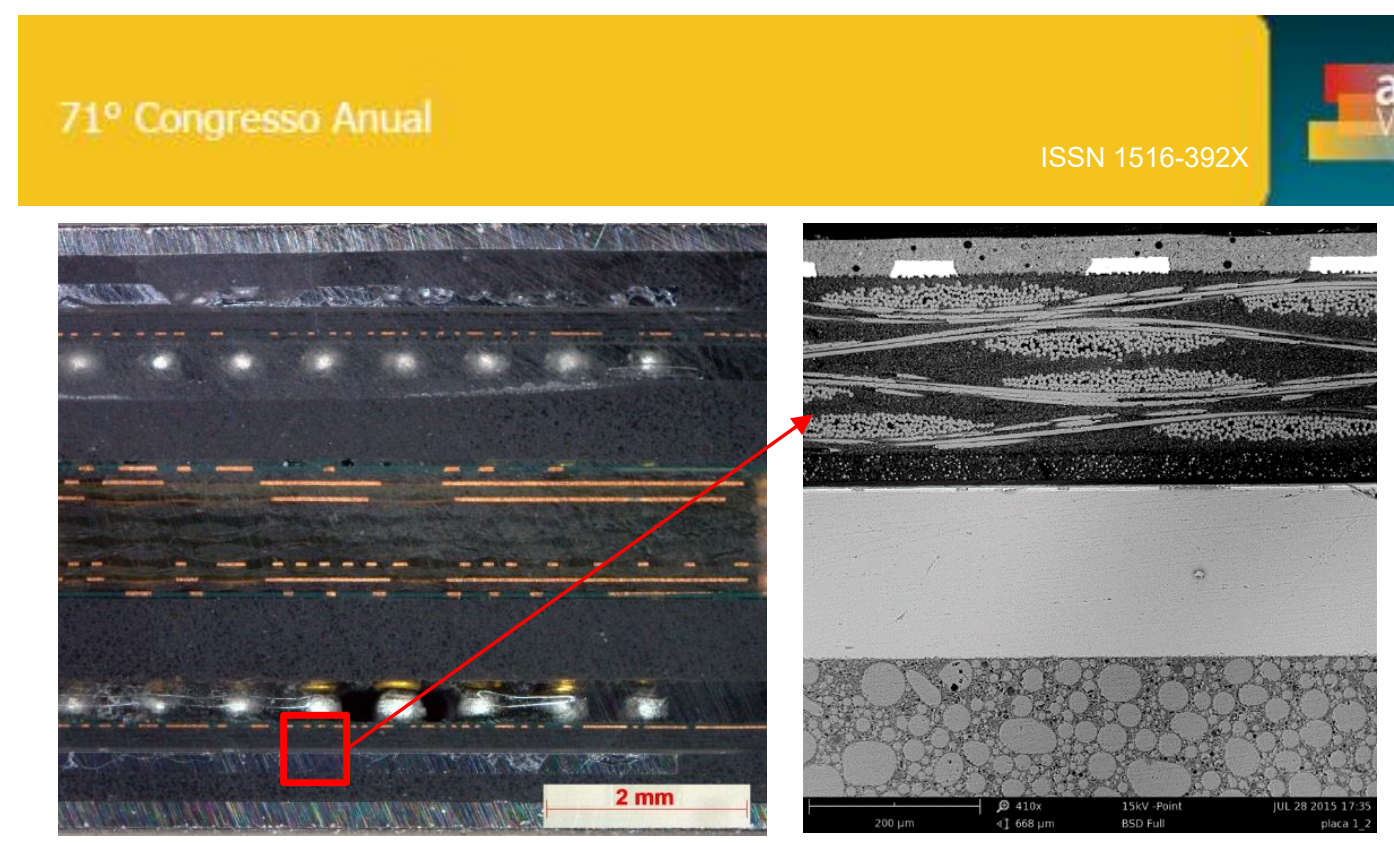

Figura 7 - Imagem de elétrons retro-espalhados da amostragem $\mathrm{C}$ da placa de memória. Á esquerda, detalhe mostrando a região em que a micrografia foi analisada.

Foi realizado o mapeamento da área detalhada da Figura 7 para verificar os elementos presentes em sua composição. A Figura 8 apresenta o mapeamento da área detalhada.

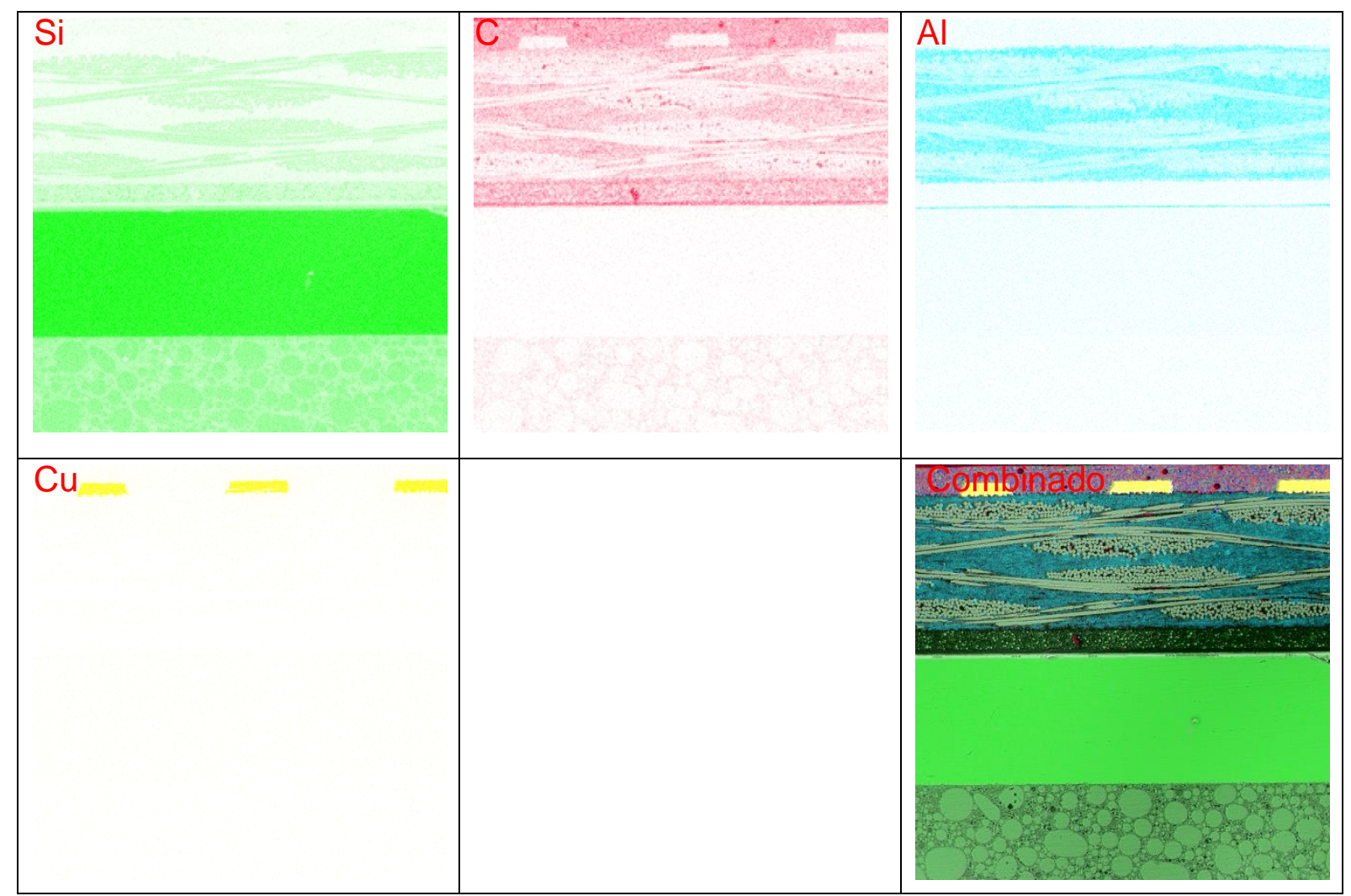

Figura 8 - Imagens de EDX da placa de memória, amostragem C

Pelo mapeamento apresentado na Figura 8 da amostragem $C$ da placa PCl-mem, pode-se ver que o circuito integrado presente na placa de memória possui uma camada de cobre e seu substrato é composto por fibra de vidro e uma resina. Assim como nas PCls a fibra de vidro é formada de silício e cálcio e a resina presente entre as fibras de vidro é composta basicamente de alumínio. 


\section{CONCLUSÃO}

A partir dos resultados obtidos até esta fase do trabalho, pode-se concluir que:

- A placa de memória é do tipo multi-layer e possui 6 camadas de cobre intercaladas por fibra de vidro e resina

- A fibra de vidro da placa de memória é composta de silício e cálcio.

- A resina da placa é composta de alumínio e carbono

- As trilhas são de cobre e a solda é composta de estanho, não apresentando chumbo em sua composição (solda lead free).

- Entre a solda e as trilhas de cobre existe uma camada de níquel utilizado como uma barreira na metalização Au/Cu.

\section{Agradecimentos}

Instituto Federal do Espírito Santo - Campus Serra

Departamento de Engenharia Química - Escola Politécnica - Universidade de São Paulo.

\section{REFERÊNCIAS}

1 SIREE Cidades Sustentáveis e Experiências Inovadoras na Gestão de Resíduos de Equipamentos Eletroeletrônicos. III Seminário Internacional Sobre Resíduos de Equipamentos Eletroeletrônicos. Recife., 2013.

2 Schluep, M.; Hagelueken, C.; et al. Recycling from e-waste to resources. 2009.

3 BERTUOL, D.A. et al. Resíduos urbanos tecnológicos: desafios e tendências. Revista Metalurgia e Metais, v. 61, p. 167-170, 2005.

4 Yamane, L.H. Associação Brasileira de Metalurgia e Materiais. p. 50-56, 2013.

5 Moraes, V.T. de Reciclagem de placas de circuito impresso de aparelhos celulares obsoletos. 2011.

6 Balakrishnan Ramesh Babu; Anand Kuber Parande; et al. Electrical and electronic waste: a global environmental problem. Waste Management \&amp; Research, v. 25, n. 4, p. 307-318, 2007.

7 Luppi, T.V. de S. Recuperação de prata a partir de radiografias. 3o Seminário de Tecnologia e Pesquisas Ambientais / SENAI, 2008.

8 Methods, S. Estudo De Impactos Ambientais Causados Por Metais Pesados Em Água Do Mar Na Baía De São Marcos : Correlações e Níveis Background. Universidade Federal da Paraíba, 2007.

9 Agricultura, C.D.E.P.E.M.; Metálica, D.E.S.; et al. Teor de metais pesados em solo contaminado com resíduo de sucata metálica, em função de sua acidificação. p. 86, 2009.

10 Gurung, M.; Adhikari, B.B.; et al. Recovery of gold and silver from spent mobile phones by means of acidothiourea leaching followed by adsorption using biosorbent prepared from persimmon tannin. Hydrometallurgy, v. 133, p. 84-93, 2013.

11 Guo, J.; Rao, Q.; et al. Application of glass-nonmetals of waste printed circuit boards to produce phenolic moulding compound. Journal of Hazardous Materials, v. 153, n. 1-2, p. 728-734, 2008.

$12 \mathrm{He}, \mathrm{W} . ;$ Li, G.; et al. WEEE recovery strategies and the WEEE treatment status in China. Journal of Hazardous Materials, v. 136, n. 3, p. 502-512, 2006.

$13 \mathrm{Li}, \mathrm{J}$;; Lu, $\mathrm{H}$.; et al. Recycle technology for recovering resources and products from waste printed circuit boards. Environmental Science and Technology, v. 41, n. 6, p. 1995-2000, 2007.

14 Naseri Joda, N.; Rashchi, F. Recovery of ultra fine grained silver and copper from PC board scraps. Separation and Purification Technology, v. 92, p. 36-42, 2012. 
15 Petter, P.M.H.; Veit, H.M.; et al. Evaluation of gold and silver leaching from printed circuit board of cellphones. Waste Management, v. 34, n. 2, p. 475-482, 2014.

16 EWALD MR Equipamentos Eletroeletrônicos - Requisitos para atividade de manufatura reversa. p. 16156, 2014.

17 Das, A.; Vidyadhar, A.; et al. A novel flowsheet for the recovery of metal values from waste printed circuit boards. Resources, Conservation and Recycling, v. 53, n. 8, p. 464-469, 2009.

18 Rochat, D.; Hagelüken, C.; et al. Optimal recycling for printed wiring boards (PWBs) in India. Proceeding of the R, 2007.

19 Abrantes, R. Reciclagem de Placas de Circuito Impresso: Optimização da Operação de Processamento Físico. Intituto Superior Técnico da Universidade Técnica de Lisboa, p. 97, 2009.

20 Oguchi, M.; Sakanakura, H.; et al. Toxic metals in WEEE: Characterization and substance flow analysis in waste treatment processes. Science of the Total Environment, v. 463-464, p. 1124-1132, 2013.

21 Park, Y.J.; Fray, D.J. Recovery of high purity precious metals from printed circuit boards. J. Hazard. Mater., v. 164, p. 1152-1158, 2009.

22 Yamane, L.H.; Moraes, V.T. de; et al. Recycling of WEEE: characterization of spent printed circuit boards from mobile phones and computers. Waste management (New York, N.Y.), v. 31, n. 12, p. 2553-8, 2011.

23 Tuncuk, A.; Stazi, V.; et al. Aqueous metal recovery techniques from e-scrap: Hydrometallurgy in recycling. Minerals Engineering, v. 25, p. 28-37, 2012.

24 Yamane, L.H. Recuperação de Metais de Placas de Circuito Impresso de Computadores Obsoletos Através de Processo Biohidrometalúrgico. p. 124, 2012.

25 Alam, M.O.; Chan, Y.C.; et al. Reliability study of the electroless Ni-P layer against solder alloy. Microelectronics Reliability, v. 42, n. 7, p. 1065-1073, 2002.

2626 Tu, P.L.; Chan, Y.C.; et al. Growth kinetics of intermetallic compounds in chip scale package solder joint. Scripta Materialia, v. 44, n. 2, p. 317-323, 2001. 